\title{
A Two-Dimensional MoS2 Catalysis Transistor by Solid-State lon Gating Manipulation and Adjustment (SIGMA)
}

Wu, Yecun; Ringe, Stefan; Wu, Chun-Lan; Chen, Wei; Yang, Ankun; Chen, Hao; Tang, Michael; Zhou, Guangmin; Hwang, Harold Y.; Chan, Karen

Total number of authors:

11

Published in:

Nano Letters

Link to article, DOI:

10.1021/acs.nanolett.9b02888

Publication date:

2019

Document Version

Peer reviewed version

Link back to DTU Orbit

Citation (APA):

Wu, Y., Ringe, S., Wu, C-L., Chen, W., Yang, A., Chen, H., Tang, M., Zhou, G., Hwang, H. Y., Chan, K., \& Cui, Y. (2019). A Two-Dimensional MoS Catalysis Transistor by Solid-State lon Gating Manipulation and Adjustment (SIGMA). Nano Letters, ${ }^{2}$ 19(10), 7293-7300. https://doi.org/10.1021/acs.nanolett.9b02888

\section{General rights}

Copyright and moral rights for the publications made accessible in the public portal are retained by the authors and/or other copyright owners and it is a condition of accessing publications that users recognise and abide by the legal requirements associated with these rights.

- Users may download and print one copy of any publication from the public portal for the purpose of private study or research.

- You may not further distribute the material or use it for any profit-making activity or commercial gain

- You may freely distribute the URL identifying the publication in the public portal 


\section{A Two-Dimensional $\mathrm{MoS}_{2}$ Catalysis Transistor by Solid-State Ion Gating Manipulation and Adjustment (SIGMA)}

Yecun Wu, ${ }^{\dagger, \nabla}$ Stefan Ringe, ${ }^{\ddagger, \S, \nabla}$ Chun-Lan Wu," Wei Chen," Ankun Yang," Hao Chen," Michael Tang, ${ }^{\ddagger, \S}$ Guangmin Zhou," Harold Y. Hwang, ${ }^{\perp, \#}$ Karen Chan, , ${ }^{*, ~ Y i ~ C u i ~}{ }^{*, \|, \#}$

${ }^{\dagger}$ Department of Electrical Engineering, Stanford University, Stanford, CA, USA.

${ }^{\ddagger}$ SUNCAT Center for Interface Science and Catalysis, Stanford University, Stanford, CA, USA.

§SUNCAT Center for Interface Science and Catalysis, SLAC National Accelerator Laboratory, Menlo Park, California, 94025, United States

"Department of Materials Science and Engineering, Stanford University, Stanford, CA, USA.

${ }^{\perp}$ Department of Applied Physics, Stanford University, Stanford, CA, USA

\#Stanford Institute for Materials and Energy Science, SLAC National Accelerator Laboratory, Menlo Park, CA, USA.

${ }^{\circledR}$ CatTheory Center, Department of Physics, Technical University of Denmark, Kongens Lyngby 2800, Denmark

These authors contributed equally to this work.

ABSTRACT: A variety of methods including tuning chemical compositions, structures, crystallinity, defects and strain and electrochemical intercalation have been demonstrated to enhance the catalytic activity. However, none of these tuning methods provide direct dynamical control during catalytic reactions. Here we propose a new method to tune the activity of catalysts 
through Solid-state Ion Gating Manipulation and Adjustment (SIGMA) using a catalysis transistor. SIGMA can electrostatically dope the surface of catalysts with a high electron concentration over $5 \times 10^{13} \mathrm{~cm}^{-2}$, and thus modulate both the chemical potential of the reaction intermediates and their electrical conductivity. The hydrogen evolution reaction (HER) on both pristine and defective $\mathrm{MoS}_{2}$ were investigated as model reactions. Our theoretical and experimental results show that the overpotential at $10 \mathrm{~mA} / \mathrm{cm}^{2}$ and Tafel slope can be in-situ, continuously, dynamically, and reversibly tuned over $100 \mathrm{mV}$ and around $100 \mathrm{mV} /$ dec respectively.

KEYWORDS: catalysis transistor, solid-state ion gating, electrocatalysis, two-dimensional materials

TOC Graphic:
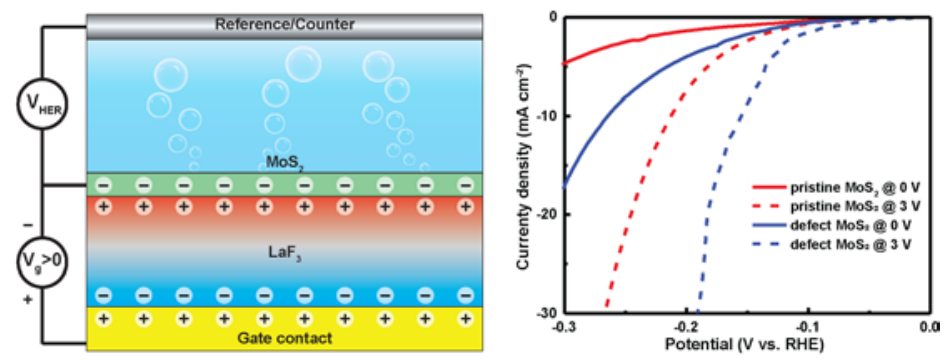

Electrocatalysis plays an important role in the conversion of earth-abundant and low-cost resources $\left(\mathrm{H}_{2} \mathrm{O}, \mathrm{CO}_{2}\right.$, etc.) to high-value products $\left(\mathrm{H}_{2}, \mathrm{CH}_{4}\right.$, etc.), especially when coupled with renewable energy ${ }^{1}$. To achieve the goal of highly efficient energy conversion at a low cost, different strategies have been proposed to tune and enhance the catalytic performance of electrocatalysts. The most general methodology is to change the chemical compositions and structure of catalysts to tune the energetics of the reaction intermediates involved ${ }^{2-4}$. Tuning the surface morphology, such as the dominant surface facet, the crystallinity, or the amount of defects in the catalyst is another popular way to improve the electrochemical conversion performance ${ }^{5-7}$. A lithium electrochemical 
intercalation tuning approach was developed to optimize the activity by varying the electron structure of existing materials ${ }^{8}$. Straining the catalysts through volume expansion/contraction is another effective technique to tune the catalytic performance in a continuous manner ${ }^{9,10}$. Despite the exciting progress, none of these strategies allows for in-situ dynamic control over the activity during the reactions. Here, we demonstrate that Solid-state Ion Gating Manipulation and Adjustment (SIGMA) as an effective method to realize an in-situ, dynamic, continuous, and reversible tuning of catalytic activity. SIGMA can manipulate the carrier density of the catalyst, which allows us to optimize the reaction intermediate binding energy and the electrical conductivity to obtain a high-performance catalyst.

Electrostatic gating has been widely used for decades for cutting-edge electronics and photonics 11-13 and extended to a variety of applications ${ }^{14-16}$. However, the relatively small capacitance of traditional gate dielectrics (for example, $\sim 10^{-2} \mu \mathrm{F} \mathrm{cm}{ }^{-2}$ for $300 \mathrm{~nm} \mathrm{SiO} 2$ ) results in only a low doping concentration $\left(\sim 10^{12} \mathrm{~cm}^{-2}\right)$ even at high gate voltage ( $\left.>50 \mathrm{~V}\right)$. Reducing the thickness of dielectrics and increasing the dielectric constant are a direction to improve, but there is a limit due to the leakage current. The electric-double-layer transistor (EDLT) with ionic liquid electrolyte offered over two orders of magnitude higher capacitance coupling $\left.(\sim 7 \mu \mathrm{F} \mathrm{cm})^{-2}\right)$ and has been successfully used in field effect transistors for accumulating carriers over $10^{14} \mathrm{~cm}^{-2}{ }^{17}$. However, it is difficult to apply ionic liquid EDLT to tune electrochemical reactions, especially for liquid phase reactions because there would be a mixing compatibility issue between the reactant electrolyte and ionic gating liquid. 
Recently, we have developed a solid-state ion gating method with a similar capacitive coupling strength $\left(\sim 4 \mu \mathrm{F} \mathrm{cm}^{-2}\right)$ as the ionic liquid to induce an insulator-metal transition in $\mathrm{MoS}_{2}$ transistors by mobile fluoride $\left(\mathrm{F}^{-}\right)$ions in $\mathrm{LaF}_{3}{ }^{18}$. Here we exploit $\mathrm{F}^{-}$ion solid-ion gating for the first time as the basis of SIGMA for catalyst tuning, which not only maintains high capacitance coupling but also removes the problem of incompatibility of liquid electrolyte mixing. Figure 1a shows our basic device structure, which can be considered as two types of devices stacked together: A) The top half is an electrocatalytic cell, where a thin layer $\mathrm{MoS}_{2}$ for hydrogen evolution reaction and a counter electrode for oxygen evolution reaction are in contact with aqueous electrolyte. B) The bottom half is a solid ion EDL transistor, where the same $\mathrm{MoS}_{2}$ layer and the gate electrode are coupled through the $\mathrm{LaF}_{3}$ solid ion conductor. When applying a positive gate voltage in the bottom cell (Figure 1b), the $\mathrm{F}^{-}$ions migrate and accumulate close to the bottom gate contact by electrostatic attraction, resulting in fluoride vacancies (positive charge) at the upper surface of the $\mathrm{LaF}_{3}$. The $\mathrm{MoS}_{2}$ layer on top of the $\mathrm{LaF}_{3}$ would be negatively doped (n-doped) to balance the charge (Figure 1b). A negative gate voltage generates the opposite effect (Figure 1c). By using this method, the $\mathrm{MoS}_{2}$ layer can be electron- doped with a concentration of $5 \times 10^{13} \mathrm{~cm}^{-2}$ when applying a gate voltage of $+3 \mathrm{~V}^{18}$. We hypothesize that such a strong gate coupling in this new SIGMA method not only changes the conductivity of catalysts but also modulates the chemical potential of the reaction intermediates (as shown schematically in free energy diagrams in the top part of Figure 1 a to c), resulting in significant catalytic activity tuning (shown in our joint theoretical and experimental study below) ${ }^{7,19}$. 
a
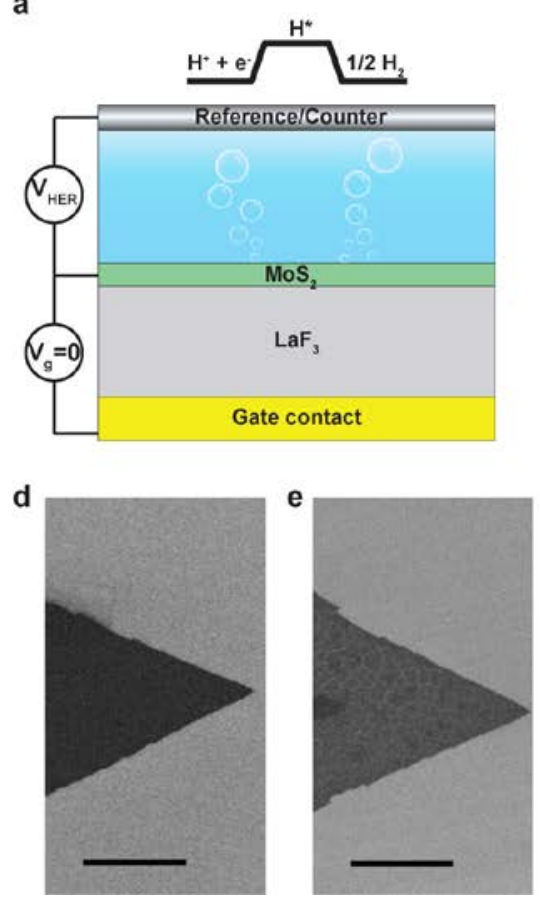

b
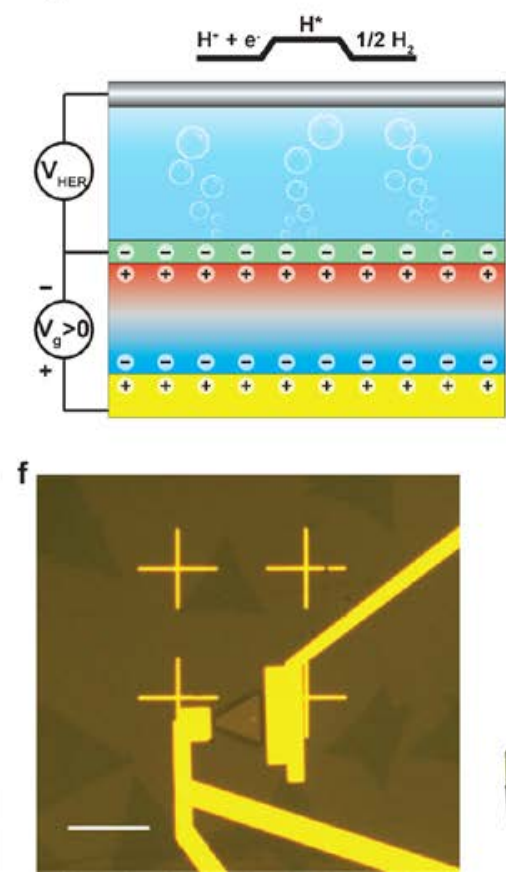

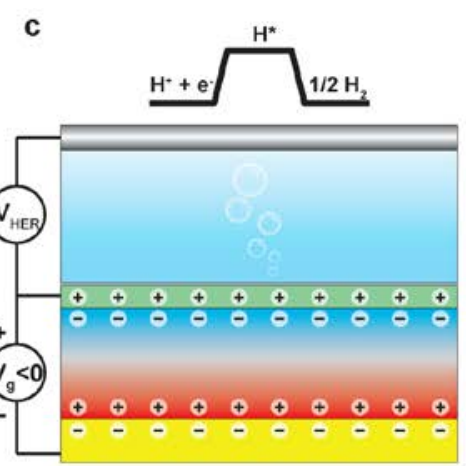

g

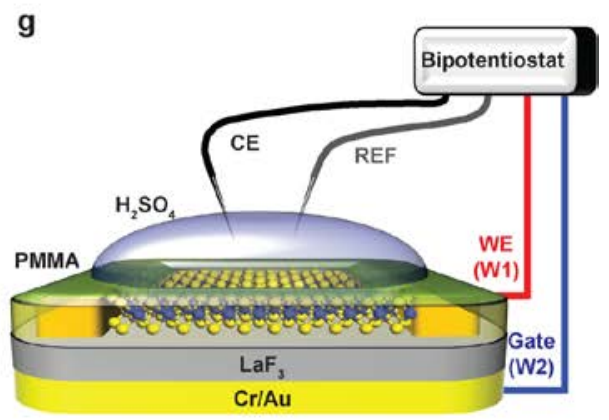

Figure 1. SIGMA for hydrogen evolution reaction. (a) Schematic illustration of the SIGMA approach and HER at zero gate voltage. SIGMA is applied to the bottom of the $\mathrm{MoS}_{2}$ and HER occurs on the top of $\mathrm{MoS}_{2}$. The free energy versus the reaction coordinate of HER for $\mathrm{MoS}_{2}$ is illustrated on top. (b) A positive gate voltage dopes the $\mathrm{MoS}_{2}$ with electrons, which enhances conductivity of $\mathrm{MoS}_{2}$ and reduces the hydrogen adsorption energy, thus enhancing the catalytic performance. (c) A negative gate voltage dopes the $\mathrm{MoS}_{2}$ with holes, thus increases the hydrogen adsorption energy. (d) SEM image of CVD grown monolayer pristine $\mathrm{MoS}_{2}$ (scale bar: $10 \mu \mathrm{m}$ ). (e) SEM image of defective CVD grown $\mathrm{MoS}_{2}$ generated by Ar plasma etching (scale bar: $10 \mu \mathrm{m}$ ). (f) Optical microscope image of the microcell, where the edge of the $\mathrm{MoS}_{2}$ is covered by PMMA and the basal plane is exposed for HER (scale bar: $50 \mu \mathrm{m}$ ). (g) Schematic illustration of the experimental setup: a drop of $0.5 \mathrm{M} \mathrm{H}_{2} \mathrm{SO}_{4}$ is placed on top of the microcell for HER; a bipotentiostat is used to form a three-electrode electrochemical measurement and gate control.

To demonstrate the SIGMA methodology, we select the $\mathrm{MoS}_{2}$ catalyzed hydrogen evolution reaction (HER) as a model system. In recent years, due to its catalytic activity, low-cost, and earthabundance, two-dimensional $\mathrm{MoS}_{2}$ has been regarded as an alternative HER catalyst material to precious metals ${ }^{20,21}$. Additionally, the low out-of-plane and high in-plane dielectric constant of the ultrathin layer make two-dimensional $\mathrm{MoS}_{2}$ highly desirable for well-controlled electrostatics ${ }^{22}$. However, the inert basal plane and the high electronic resistance of $2 \mathrm{H}-\mathrm{MoS}_{2}$ greatly limit their catalytic performance ${ }^{23,24}$. Intensive studies have been carried out to increase the density of active 
sites ${ }^{25}$, showing that the HER performance of $\mathrm{MoS}_{2}$ can be improved by exposing more edges ${ }^{26}$, ${ }^{27}$, introducing dopants ${ }^{3,28,29}$, or creating sulfur vacancies (defective $\mathrm{MoS}_{2}$ ) as active sites ${ }^{6,7,30}$.

In what follows, we show that the HER catalytic activity of both pristine and defective $\mathrm{MoS}_{2}$ devices can be tuned by SIGMA. We find that SIGMA can dynamically, continuously, and reversibly tune the hydrogen adsorption free energy ( $\Delta G_{\text {ads }}$ ) and the conductivity of $\mathrm{MoS}_{2}$, thus tuning the performance of a catalyst. The overpotential at $10 \mathrm{~mA} / \mathrm{cm}^{2}$ can be decreased by over $100 \mathrm{mV}$ in both pristine and defective $\mathrm{MoS}_{2}$. SIGMA opens up a new way to dynamically tune the catalytic conversion efficiency independently of the applied potential and without direct modification of the catalyst material or morphology.

\section{Results and discussions}

To investigate the gate effect on the HER of a single layer $\mathrm{MoS}_{2}$ crystal, we first grew the triangular monolayer $\mathrm{MoS}_{2}$ by chemical vapor deposition (CVD) on a silicon substrate with $300 \mathrm{~nm}$ oxide layer as shown in the Figure S1. The defective $\mathrm{MoS}_{2}$ was fabricated by using mild argon plasma etch of the pristine $\mathrm{MoS}_{2}$. The SEM images of the pristine and defective $\mathrm{MoS}_{2}$ are shown in Figure $1 \mathbf{d}$ and e. Defective $\mathrm{MoS}_{2}$ shows short and isolated cracks with a connection angle of $\sim 120^{\circ}$, which is consistent with the previous report ${ }^{6}$. Raman spectra, photoluminescence (PL), and atomic force microscope (AFM) measurements were used to confirm the formation of single layer $\mathrm{MoS}_{2}$ (Figure S2 and S3). X-ray photoelectron spectroscopy (XPS) was employed to measure the ratio of Mo and S, which is used to determine the density of sulfur vacancies (Figure S4). The Mo and S ratio of the defective samples is 1:1.75 $( \pm 0.05)$, indicating that the sulfur vacancy density is $\sim 12.5 \%$ ( $\pm 3 \%$ ) (Figure S4). We then transferred the as-grown and plasma treated samples to a 
$\mathrm{LaF}_{3}$ substrate by the polymer-assisted wet transfer method. After the transfer, the sample was annealed in argon atmosphere at $250{ }^{\circ} \mathrm{C}$ to remove the contaminants and achieve a high quality $\mathrm{LaF}_{3}-\mathrm{MoS}_{2}$ contact interface. Subsequently, electron-beam lithography and metal evaporation were used to define and deposit $5 \mathrm{~nm}$ of chromium as an adhesion layer and $45 \mathrm{~nm}$ gold electrodes as current collectors near the materials. To achieve a low contact resistance, we also fabricated a $50 \mathrm{~nm}$ pure Au electrode as a connection between the $\mathrm{MoS}_{2}$ flake and $\mathrm{Cr} / \mathrm{Au}$ electrodes. Finally, a layer of 5/50 nm Cr/Au was deposited by e-beam evaporation on the back side of the $\mathrm{LaF}_{3}$ to form a back-gate structure. The schematic of a $\mathrm{MoS}_{2}$ solid state EDL transistor is shown in Figure S5a.

To investigate the electron transport properties in pristine and defective $\mathrm{MoS}_{2}$, we first performed electrical characterization of our EDL transistors. All the transistors are fabricated with the same channel length and width, and the measurements were performed at room temperature using a semiconductor parameter analyzer with a shielded probe station. The voltage sources connected in the configuration are depicted in the Figure S5a. A 2 V constant source-drain voltage was applied and the bottom ionic gate voltage was swept from $-1 \mathrm{~V}$ to $3 \mathrm{~V}$ with $10 \mathrm{mV} / \mathrm{s}$ step, while the sourcedrain current was measured. The gating responses of $\mathrm{MoS}_{2}$ and defective $\mathrm{MoS}_{2}$ channel are presented in Figure S5b. Repeated sweeps on the same device did not show significant variation and the leakage is lower than $20 \mathrm{nA}$. The pristine $\mathrm{MoS}_{2}$ device demonstrates a typical n-type channel with a threshold voltage and on/off ratio over $10^{4}$. The source-drain current shows a tendency of saturation when the gate voltage is greater than $2.5 \mathrm{~V}$. The carrier density of the EDL transistor can be estimated by using $n=(C \times V) / q$, where the $C$ is the capacitance of the solid-state $\mathrm{MoS}_{2}-\mathrm{LaF}_{3}$ electrical double layer $\left(\sim 4 \mu \mathrm{F} / \mathrm{cm}^{2}\right)^{18}, \mathrm{~V}$ is the gate voltage, and $\mathrm{q}$ is the charge of an electron $\left(1.6 \times 10^{-19} \mathrm{C}\right)$. At $3 \mathrm{~V}$ gate voltage, the carrier density is estimated to be over $5 \times 10^{13}$ 
$\mathrm{cm}^{-2}$. As for the defective $\mathrm{MoS}_{2}$ channel, limited charge transport was observed. A higher threshold voltage was obtained, and the current at $0 \mathrm{~V}$ gate voltage is about one order magnitude lower than that of the pristine $\mathrm{MoS}_{2}$ channel. This charge transport limit in the subthreshold regime origins from the trap states induced by the defects in the band gap of $\mathrm{MoS}_{2}{ }^{31}$. Despite of the current decline at $0 \mathrm{~V}$, the current at higher electron doping concentration is higher than that of the pristine $\mathrm{MoS}_{2}$ channel device. The in-situ Raman spectra of the gate dependent defective $\mathrm{MoS}_{2}$ were conducted to confirm that there is no $2 \mathrm{H}$ to $1 \mathrm{~T}$ phase transition at higher gate bias (Figure S6).
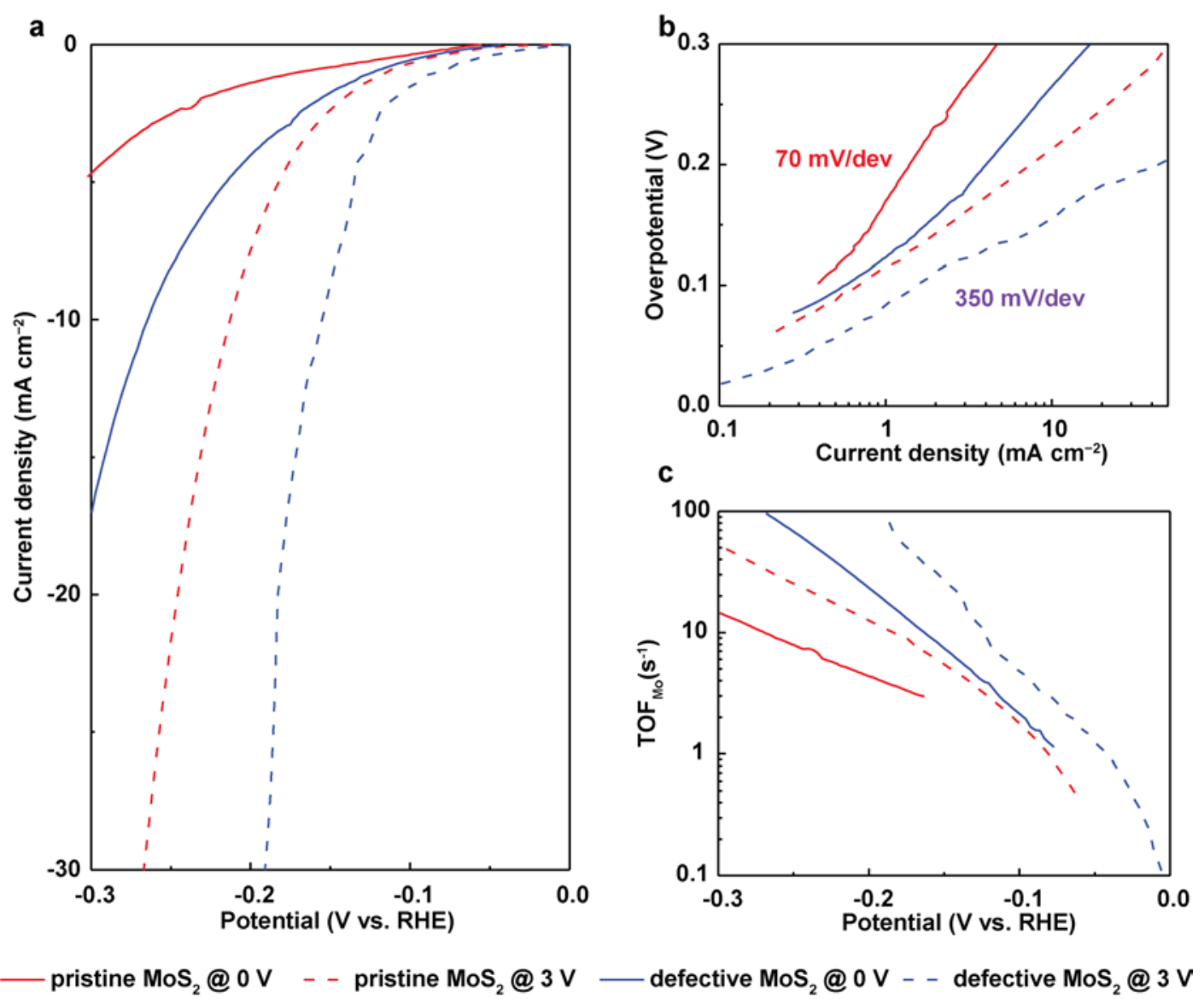

Figure 2. Electrochemical tuning of the $\mathrm{MoS}_{2}$ HER by SIGMA. (a) Polarization curves measured from the pristine (red) and defective (blue) $\mathrm{MoS}_{2}$ device at $0 \mathrm{~V}$ (solid) and $3 \mathrm{~V}$ (dash) gate voltage without iR correction. (b) The corresponding Tafel plots of the polarization curves. (c) Corresponding TOF $\mathrm{Mo}$ plots of the LSV curves. 
To combine the EDL transistor and electrochemical catalysis, a layer of poly(methylmethacrylate) (PMMA) resist is spin-coated as a protection layer $(\sim 200 \mathrm{~nm})$ on top of the transistor. A window is then opened in the PMMA by electron beam lithography to cover the edge and expose the basal plane of $\mathrm{MoS}_{2}$ for HER as shown in the optical microscope image in Figure 1f. A small amount ( $\sim 30 \mu \mathrm{L})$ of the $0.5 \mathrm{M}$ sulfuric acid $\left(\mathrm{H}_{2} \mathrm{SO}_{4}\right)$ electrolyte was dropped on top of the device. An $\mathrm{Ag} / \mathrm{AgCl}$ reference electrode and a sharp carbon rod counter electrode were immersed in the electrolyte to form a three-electrode system. The experiment was performed on a bipotentiostat as shown in Figure 1g. The equivalent circuit can be found in Figure S7. We also used the $0.5 \mathrm{M}$ $\mathrm{H}_{2} \mathrm{SO}_{4}$ electrolyte as a top gate of the device to confirm that the $\mathrm{MoS}_{2}$ cannot be gated by the HER electrolyte. The results showed negligible changes of the conductivity of $\mathrm{MoS}_{2}$ with the top $0.5 \mathrm{M}$ $\mathrm{H}_{2} \mathrm{SO}_{4}$ gate (Figure $\mathrm{S} 8$ ).

Linear sweep voltammograms (LSV) were performed on both pristine $\mathrm{MoS}_{2}$ and defective $\mathrm{MoS}_{2}$ with different gate voltages ranging from $0 \mathrm{~V}$ to $3 \mathrm{~V}$. The polarized curves of samples at $0 \mathrm{~V}$ and $3 \mathrm{~V}$ gate voltages without iR correction in are shown in Figure 2a. The corresponding Tafel slope curves are presented in Figure $2 \mathbf{b}$. Completely polarized curves from $0 \mathrm{~V}$ to $3 \mathrm{~V}$ gate voltage with $1 \mathrm{~V}$ steps are shown in the Figure S9. Electrocatalysis from monolayer single crystal $\mathrm{MoS}_{2}$ also allows an accurate measurement of the surface exposed to the electrolyte, which can be used to calculate the turnover frequency (TOF), an indicator of the activity per single catalytic site. The TOFs of each individual S-atom are calculated and shown in Figure 2c to compare the intrinsic activity of different $\mathrm{MoS}_{2}$ samples. To investigate the correlation between the HER catalytic performance and gate voltage, we fabricated multiple devices and measured the catalytic performance at different gate voltages to obtain statistical data. 
The variation of the overpotential at $10 \mathrm{~mA} / \mathrm{cm}^{2}$ and the Tafel slopes of both pristine $\mathrm{MoS}_{2}$ and defective $\mathrm{MoS}_{2}$ with different gate voltages are summarized in Figure $\mathbf{3}$ a and $\mathbf{b}$, respectively. For the pristine $\mathrm{MoS}_{2}$ device, the catalyst shows an overpotential over $300 \mathrm{mV}$ at $10 \mathrm{~mA} / \mathrm{cm}^{2}$ and a Tafel slope over $250 \mathrm{mV} / \mathrm{dec}$ at zero gate voltage. As the gate voltage increases to $3 \mathrm{~V}$, this situation is dramatically improved, with the overpotential at $10 \mathrm{~mA} / \mathrm{cm}^{2}$ reduced to $\sim 210 \mathrm{mV}$. At $3 \mathrm{~V}$ gate voltage, the Tafel slope is reduced to $110 \mathrm{mV} / \mathrm{dec}$ which has been associated with a Volmer-Heyrovsky mechanism with the adsorption Volmer step $\left(\mathrm{H}^{+}+\mathrm{e}^{-}+* \rightarrow \mathrm{H}^{*}\right)$ being the rate-limiting step ${ }^{26}$. The decrease in Tafel slope with gate voltage could be rationalized from the gate-induced electron injection into the *H@MoS 2 system. From our DFT calculations and Bader charge partitioning analysis ${ }^{32}$, we found this to also negatively charge up the adsorbed $* \mathrm{H}$ atoms. We postulate here that this increased electronegativity of ${ }^{*} \mathrm{H}$ then leads to a more final-state like transition state, which increases the charge transfer coefficient and reduces the Tafel slope.

The defective $\mathrm{MoS}_{2}$ is much more intrinsically active for HER than the pristine case. By increasing the gate voltage, we find a similar, almost linear reduction of the overpotential and a decrease of the Tafel slope as in the pristine case. Both the pristine and the defective systems can thus be activated to a comparable degree by applying the gate voltage. Additionally, the increased conductivity also helps to reduce the iR loss in electron transport in $\mathrm{MoS}_{2}$ and between $\mathrm{MoS}_{2}$ and current collector $^{19}$, which contributes to the enhanced performance.

To demonstrate the reversibility and in-situ tuning capability of our SIGMA method, a "catalysis transistor" was tested as shown in Figure 3c. The HER voltage between the working and reference 
electrodes was kept constant $\left(\mathrm{V}_{\mathrm{HER}}=-0.2 \mathrm{~V}\right)$ and the gate voltage was swept from $0 \mathrm{~V}$ to $3 \mathrm{~V}$ with a $10 \mathrm{mV} / \mathrm{s}$ step. This type of test is similar to the $\mathrm{I}_{\mathrm{d}}-\mathrm{V}_{\mathrm{g}}$ transfer curve in a transistor to characterize the gate induced on/off states. For both the pristine and defective samples, the HER currents increase with the gate voltage, which makes it behave as an n-type channel transistor. The similar on/off ratio of $\sim 7$ of the pristine and defective device is obtained, indicating a similar underlying mechanism which will be discussed later. The gating effect is reversible with hysteresis. The hysteresis of catalysis transistor is larger than that of the $\mathrm{MoS}_{2}-\mathrm{LaF}_{3} \mathrm{EDLT}{ }^{18}$. The enhanced hysteresis could result from the aqueous environment of the catalysis transistor. Previous researches have reported that water molecular phyisorbe on the $\mathrm{MoS}_{2}$ surface facilitate positive charge trapping and detrapping at the trap levels ${ }^{33,34}$. Additionally, the hydrogen absorption and dissociation on the $\mathrm{MoS}_{2}$ surface contribute to the hysteresis. The hysteresis makes the HER performance depends on the gate voltage sweep range, direction and rate, however, it can be also utilized to fabricate "catalysis memory" to store information by catalytic activities.

A comparison of our SIGMA method and other reported methods is presented in Figure 3d. Existing methods give a point in the figure, while our SIGMA method shows a dynamic and continuously tunable range. The optimized performance of our method is close to the best performance from those sophisticated designs. 

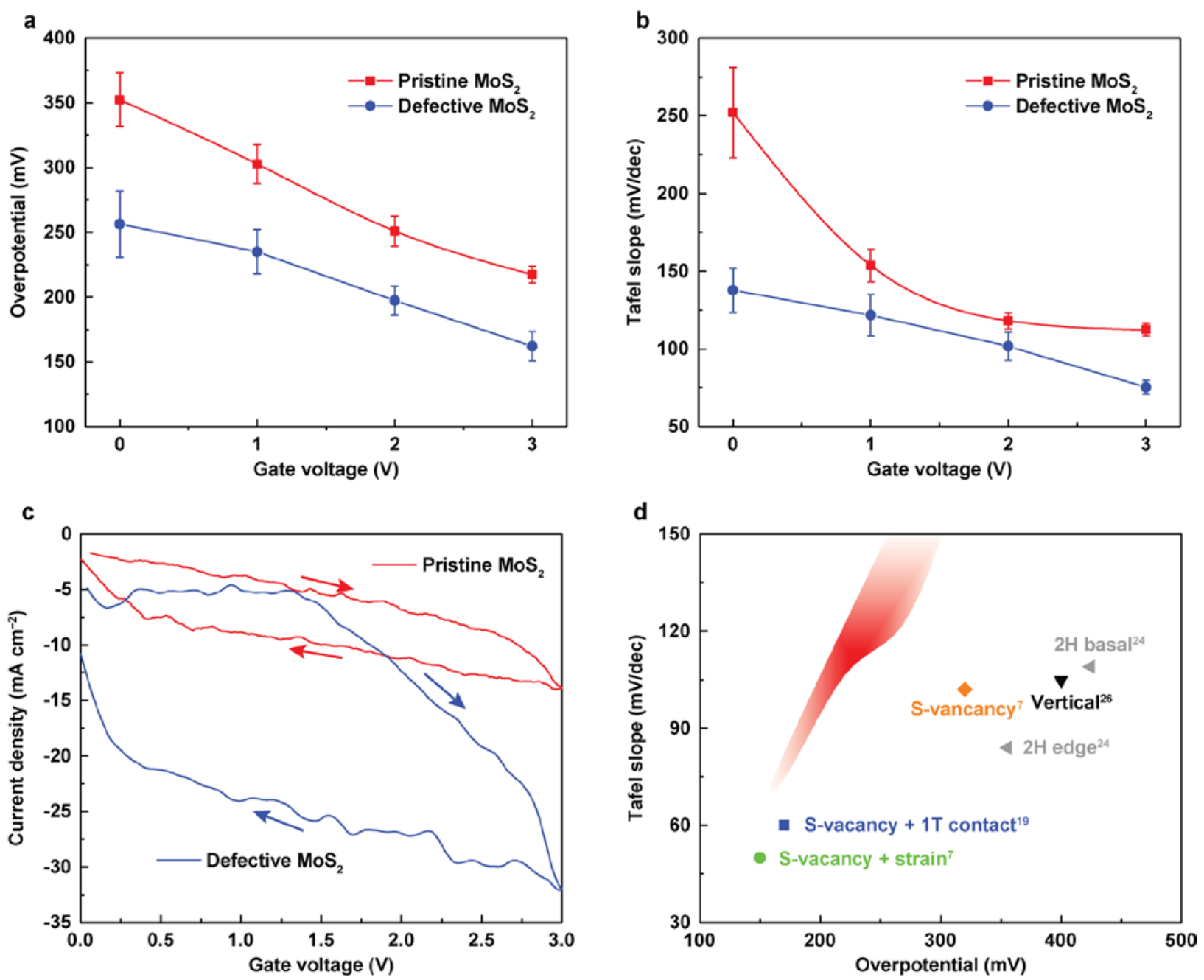

Figure 3. Gate tunable HER performance. (a) The variation of the overpotential at $10 \mathrm{mV} / \mathrm{cm}^{2}$ with gate voltage. (b) The variation of the Tafel slope with gate voltage. (c) The $\mathrm{I}_{\mathrm{HER}}-\mathrm{V}_{\mathrm{g}}$ transfer curves with $\mathrm{V}_{\mathrm{HER}}=-0.2 \mathrm{~V}$. (d) A comparison of the HER performances of CVD $\mathrm{MoS}_{2}$ modified by different methods. The boundary of the red area is the performance of pristine and defective $\mathrm{MoS}_{2}$ obtained from (a) and (b).

Tuning the gate voltage with SIGMA increases the charge carrier density, which can have a significant impact on surface electrocatalysis. In the case of HER, the overall reaction rate has been shown to be determined mainly by the ${ }^{*} \mathrm{H}$ binding energy, $\Delta G_{\mathrm{ads}}{ }^{23}$. A binding energy that is too weak $\left(\Delta G_{\text {ads }}>0\right)$ or too strong $\left(\Delta G_{\text {ads }}<0\right)$ will cause the rate to be limited by the $* \mathrm{H}$ adsorption (Volmer) step or desorption (Heyrovsky/Tafel) steps, respectively. A binding energy of $\Delta G_{\text {ads }} \approx 0$ has been determined as a characteristic of an ideal HER catalyst ${ }^{35}$. A previous study has shown 
that the basal plane should not be considered as an active site, with $3 \%$ of S-vacancies already exhibiting orders of magnitudes higher HER activity ${ }^{30}$. In our as-grown pristine $\mathrm{MoS}_{2}$, a small ratio of intrinsic atomic defects is unavoidable ${ }^{36,37}$. Beside the sulfur vacancies, the hydrogen coverage (number of $\mathrm{H}$ atoms over number of $\mathrm{S}$ atoms on the upper side of $\mathrm{MoS}_{2}$ unit cell) has been also shown to affect the activation barrier of the reaction ${ }^{38}$.

We evaluated $\Delta G_{\text {ads }}$ at varying sulfur vacancy coverages as a function of charge carrier density. Figure $4 \mathbf{b}$ shows the results of pristine ( $0 \%$ ) and $13 \%$ sulfur vacancy $\mathrm{MoS}_{2}$ under a hydrogen coverage of $16.7 \%$. The results of different levels of defects and hydrogen coverages are presented in Figure S10. We found that the added negative surface charge stabilizes * $\mathrm{H}$ in all considered cases. This finding is consistent with previous studies, which found ${ }^{*} \mathrm{H}$ binding to be stabilized in the presence of excess electrons, thereby reducing the Volmer thermochemical reaction barrier ${ }^{19}$. Thermodynamically, at the applied HER voltages, all vacancies can be expected to be covered with $\mathrm{H}$ atoms ${ }^{30}$. By introducing excess electrons into the system, under a high hydrogen coverage and high vacancy density, we found $\Delta G_{\text {ads }}$ approach a value of zero under the range of experimentally estimated carrier densities. This minimization of the thermochemical $\mathrm{H}$ adsorption barrier that we see for most considered coverages is in agreement with the experimentally observed HER overpotential reduction. Furthermore, theory predicts a similar ${ }^{*} \mathrm{H}$ binding energy change with carrier density, independent of the vacancy coverage, in line with the experimental findings. We finally also comment on the stabilization of ${ }^{*} \mathrm{H}$ to negative $\Delta G_{\text {ads }}$ values with gate voltage that we observe in the case of small vacancy concentrations (4.2\%), as shown in the supporting information. In this case one would expect a decrease the HER activity as gate voltage increases. 
This finding suggests that HER proceeds mainly from clustered S-vacancy regions, corresponding to higher local *H coverages and optimal binding energies under an applied gate voltage.

Additionally, in typical electrochemical devices, the surface charge of a catalyst affects the potential drop which drives charge-transfer kinetics at the solid-liquid interface (Frumkin effect). In the device considered here, the SIGMA induced carrier density in $\mathrm{MoS}_{2}$ is, however, fully compensated by the surface charge density of $\mathrm{LaF}_{3}$ due to its comparably high capacitance. The electric field is thus completely screened and the potential driving force is nearly independent of the applied gate voltage. However, if the gate voltage is too high (>3 V), the field effect cannot be completely screened by the $\mathrm{MoS}_{2}$ and will penetrate through the electrolyte ${ }^{39}$. The leaking field accumulates excessive electrons at the electrical double layer of solvent and $\mathrm{MoS}_{2}$, thus affecting the electrochemical potential of reactive proton donors as well as the local $\mathrm{pH}$ at the outer Helmholtz layer.

Therefore, our catalysis transistor modulates the ${ }^{*} \mathrm{H}$ binding energy ( $\Delta \mathrm{Gads}$ ) by changing the carrier density of the catalyst through the electric field, which facially manifests as the modulation of catalytic current density. This technique can be used to control the activity of catalysts and integrated with existing circuit technology for a variety of applications. For example, in the ondemand fuel production or biomimetic systems, it can be used as a key component in feedback loop to generate regulated TOF as needed dynamically. 

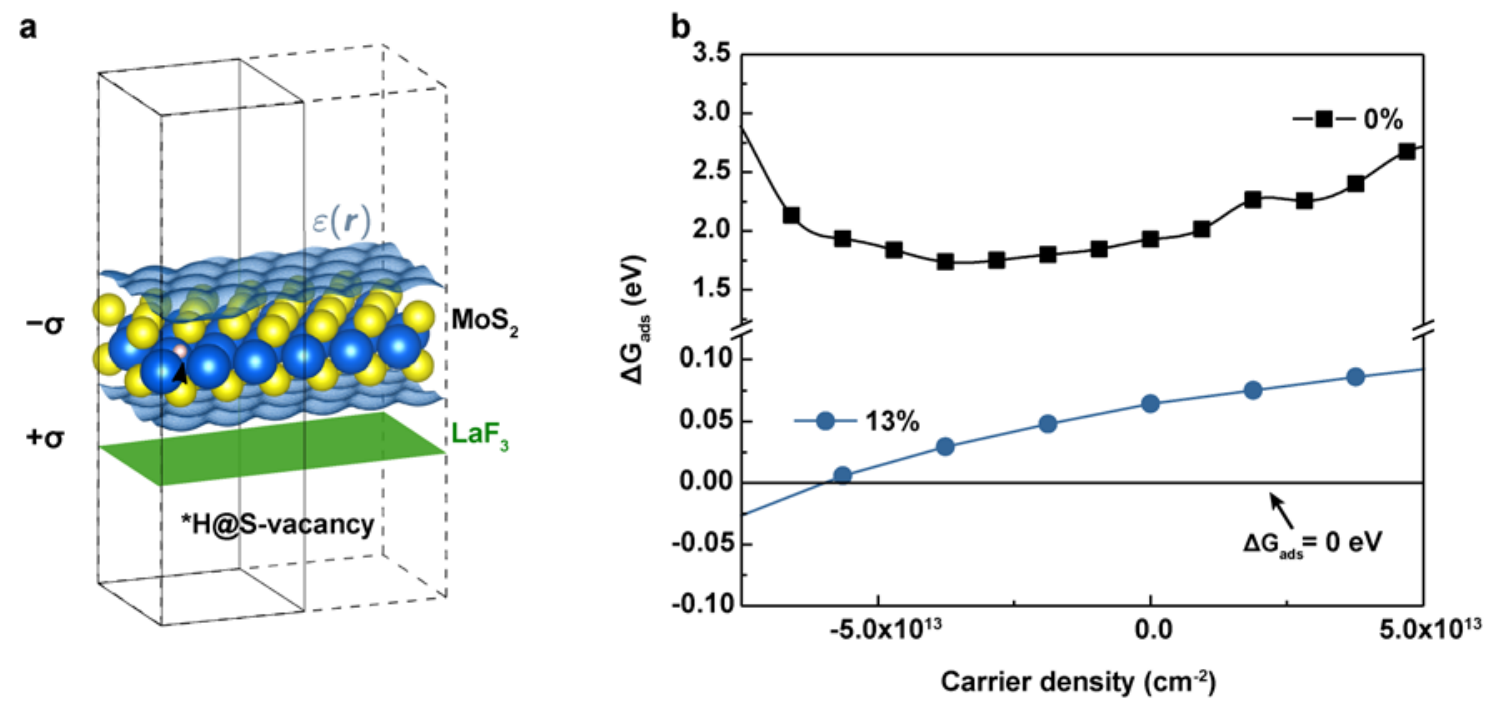

Figure 4. Computational Modeling of $\mathrm{HER}$ at the $\mathrm{MoS}_{2}$ sheet under influence of SIGMA. (a) Schematic system set-up consisting of $\mathrm{MoS}_{2}$ sheet embedded into a dielectric continuum that contains a counter charge representing the $\mathrm{LaF}_{3}-\mathrm{MoS}_{2}$ capacitor. (b) Effect of electron (negative) and hole (positive) carrier density on the adsorption energy of $\mathrm{H}^{*}$ for $0 \%$ sulfur vacancy and $13 \%$ sulfur vacancy at a hydrogen coverage of $16.7 \%$ (all S-vacancies occupied with $\mathrm{H}$ atoms).

\section{Conclusion}

In this work, we developed a new approach (Solid-state Ion Gating Manipulation and Adjustment (SIGMA)) to dynamically, continuously, and reversibly tune the catalytic performance of electrochemical devices. As for the $\mathrm{MoS}_{2}$ catalyzed HER, our experimental and theoretical results show that SIGMA tunes the carrier density of $\mathrm{MoS}_{2}$ up to $5 \times 10^{13} \mathrm{~cm}^{-2}$. These additional electrons drive the hydrogen adsorption energy to zero and increase the electronic conductivity of $\mathrm{MoS}_{2}$. SIGMA tuning of surface charge density and electric fields could represent a general strategy to tune and improve the catalytic activity without modifying structure and composition of the catalysts. In addition to fundamental studies, the catalysis transistor makes it possible to integrated the electrochemical reactions with advanced circuit technology for various on-demand applications. 


\section{Methods}

\section{CVD growth and transfer of monolayer $\mathrm{MoS}_{2}$}

Monolayer single crystal $2 \mathrm{H}-\mathrm{MoS}_{2}$ was grown in a tube furnace by CVD with molybdenum trioxide $\left(\mathrm{MoO}_{3}\right)$ and sulfur (S) powder as precursors and argon as the carrier gas. A Si substrate with $300 \mathrm{~nm}$ oxide layer was suspended face-down on a quartz boat containing the $\mathrm{MoO}_{3}$ source. The growth process was conducted at $750{ }^{\circ} \mathrm{C}$ with 5 -sccm Ar flow at atmospheric pressure and $\mathrm{Ar}$ environment. After 15 minutes growth, the furnace was cooled down to room temperature. The triangular single crystal was on the edges of the continuous film. To transfer the $\mathrm{MoS}_{2}$, a layer of PMMA was first spin-coated (4000 rpm), and the sample was baked at $120^{\circ} \mathrm{C}$ for two minutes. The diluted potassium hydroxide $(\mathrm{KOH})$ solution was then used as the etchant to etch away the $\mathrm{SiO}_{2}$ layer. After that, the PMMA/MoS 2 layer was lifted off and then transferred onto a $\mathrm{LaF}_{3}$ substrate. Finally, the sample was air-dried and the PMMA was washed off with acetone and 2propanol.

\section{Generating defective $\mathrm{MoS}_{2}$}

The argon plasma treatment was carried out by using an Oxford 80 Reactive Ion Etcher with an 8 W power supply. The gas pressure was controlled at 900 mTorr by flowing argon at $100 \mathrm{sccm}$. The $\sim 13 \%$ S-vacancies could be achieved by 20s exposure.

\section{Device fabrication}

The electron beam lithography was conducted by a JBX-6300FS Electron Beam Lithography System to pattern the electrodes. The metal evaporation process was done by a KJ Lesker egun/beam evaporator with a speed of $1 \AA /$ s and a base pressure under $10^{-6}$ Torr.

\section{Electrochemical measurements}

The electrochemical testing was performed using a three electrodes setup. A sharp carbon rod was used as a counter electrode, while a home-made $\mathrm{Ag} / \mathrm{AgCl}$ reference electrode was used as a 
reference electrode. The reference voltage of the homemade Ag/AgCl was calibrated by standard hydrogen evolution. The $\mathrm{MoS}_{2}$ flake was used as a working electrode. A drop $(\sim 30 \mu \mathrm{l})$ of $0.5 \mathrm{M}$ sulfuric acid $\left(\mathrm{H}_{2} \mathrm{SO}_{4}\right)$ solution was used as the electrolyte. The LSV scan rate was $5 \mathrm{mV} / \mathrm{s}$.

\section{DFT calculation}

Density functional theory (DFT) calculations of reaction energetics were carried out with a periodic plane-wave implementation and ultrasoft pseudopotentials using QUANTUM ESPRESSO version $6.1^{40}$ interfaced with the Atomistic Simulation Environment (ASE) ${ }^{41}$.

Acknowledgements: This work was supported by the U.S. Department of Energy (DOE), Office of Basic Energy Sciences, Division of Materials Sciences and Engineering (contract no. DEAC02-76SF00515). Part of this work was performed at the Stanford Nano Shared Facilities (SNSF), supported by the National Science Foundation under award ECCS-1542152. Computational work was supported by the U.S. Department of Energy, Chemical Sciences, Geosciences, and Biosciences (CSGB) Division of the Office of Basic Energy Sciences, via Grant DE-AC02-76SF00515 to the SUNCAT Center for Interface Science and Catalysis. K.C. acknowledges a grant (9455) from the VILLUM FONDEN.

\section{Corresponding Author}

Karen Chan, Email: kchan@fysik.dtu.dk

Yi Cui, Email: yicui@stanford.edu

\section{Notes}

The authors declare no competing financial interest. 
Author contributions: Y.W. and Y.C. conceived the idea and designed the experiments. Y.W. performed material growth, electrode fabrication, and electrochemical measurements. S.R., M.T., and K.C. carried out the theoretical calculations. C.-L.W., and A.Y. assisted with the material growth, electrode fabrication, and characterization. W.C. assisted with the electrochemical measurements. H.C. performed the SEM and XPS measurement. Y.W., S. R., and Y.C. wrote the manuscript, and all authors discussed the results and commented on the manuscript.

\section{Supporting Information:}

Figures S1 to S10

Supporting Text

Supporting References

\section{References:}

1. $\quad$ Seh, Z. W.; Kibsgaard, J.; Dickens, C. F.; Chorkendorff, I. B.; Norskov, J. K.; Jaramillo, T. F. Science 2017, 355, (6321), 146.

2. $\quad$ Kong, D.; Cha, J. J.; Wang, H.; Lee, H.; Cui, Y. Energ. Environ. Sci. 2013, 6, (12), 3553-3558.

3. Wang, H.; Tsai, C.; Kong, D.; Chan, K.; Abild-Pedersen, F.; Norskov, J.; Cui, Y. Nano Res. 2015, 8, (2), 566-575.

4. $\quad$ Zheng, X.; Ji, Y.; Tang, J.; Wang, J.; Liu, B.; Steinruck, H. G.; Lim, K.; Li, Y.; Toney, M. F.; Chan, K.; Cui, Y. Nat. Catal. 2019, 2, (1), 55-61.

5. $\quad$ Lu, Z.; Chen, G.; Li, Y.; Wang, H.; Xie, J.; Liao, L.; Liu, C.; Liu, Y.; Wu, T.; Li, Y.; Luntz, A. C.; Bajdich, M.; Cui, Y. J. Am. Chem. Soc. 2017, 139, (17), 6270-6276.

6. $\quad$ Ye, G.; Gong, Y.; Lin, J.; Li, B.; He, Y.; Pantelides, S. T.; Zhou, W.; Vajtai, R.; Ajayan, P. M. Nano Lett. 2016, 16, (2), 1097-1103.

7. Li, H.; Tsai, C.; Koh, A. L.; Cai, L.; Contryman, A. W.; Fragapane, A. H.; Zhao, J.; Han, H.; Manoharan, H. C.; Abild-Pedersen, F.; Norskov, J. K.; Zheng, X. Nat. Mater. 2016, 15, (3).

8. $\quad$ Lu, Z.; Jiang, K.; Chen, G.; Wang, H.; Cui, Y. Adv. Mater. 2018, 30, (48).

9. Wang, H.; Xu, S.; Tsai, C.; Li, Y.; Liu, C.; Zhao, J.; Liu, Y.; Yuan, H.; Abild-Pedersen, F.; Prinz, F. B.; Norskov, J. K.; Cui, Y. Science 2016, 354, (6315), 1031-1036.

10. Wang, L.; Zeng, Z. H.; Gao, W. P.; Maxson, T.; Raciti, D.; Giroux, M.; Pan, X. Q.; Wang, C.; Greeley, J. Science 2019, 363, (6429), 870-+.

11. Radisavljevic, B.; Radenovic, A.; Brivio, J.; Giacometti, V.; Kis, A. Nat. Nanotechnol. 2011, 6, (3), 147-150.

12. $\quad$ Liu, Y.; Duan, X.; Huang, Y.; Duan, X. Chem. Soc. Rev. 2018, 47, (16), 6388-6409.

13. Mak, K. F.; Shan, J. Nat. Photonics 2016, 10, (4), 216-226.

14. Sood, A.; Xiong, F.; Chen, S.; Wang, H.; Selli, D.; Zhang, J.; McClellan, C. J.; Sun, J.; Donadio, D.; Cui, Y.; Pop, E.; Goodson, K. E. Nat. Commun. 2018, 9, 4510.

15. Wang, J.; Yan, M.; Zhao, K.; Liao, X.; Wang, P.; Pan, X.; Yang, W.; Mai, L. Adv Mater 2017, 29, (7). 
16. $\quad$ Deng, Y.; Yu, Y.; Song, Y.; Zhang, J.; Wang, N. Z.; Sun, Z.; Yi, Y.; Wu, Y. Z.; Wu, S.; Zhu, J.; Wang, J.; Chen, X. H.; Zhang, Y. Nature 2018, 563, (7729), 94-99.

17. Zhang, Y.; Ye, J.; Matsuhashi, Y.; Iwasa, Y. Nano Lett 2012, 12, (3), 1136-40.

18. Wu, C.-L.; Yuan, H.; Li, Y.; Gong, Y.; Hwang, H. Y.; Cui, Y. Nano Lett 2018, 18, (4), 2387-2392.

19. Voiry, D.; Fullon, R.; Yang, J.; Silva, C. D. C. E.; Kappera, R.; Bozkurt, I.; Kaplan, D.; Lagos, M.

J.; Batson, P. E.; Gupta, G.; Mohite, A. D.; Dong, L.; Er, D.; Shenoy, V. B.; Asefa, T.; Chhowalla, M. Nat. Mater. 2016, 15, (9), 1003-1009.

20. Ding, Q.; Song, B.; Xu, P.; Jin, S. Chem 2016, 1, (5), 699-726.

21. Deng, D.; Novoselov, K. S.; Fu, Q.; Zheng, N.; Tian, Z.; Bao, X. Nat. Nanotechnol. 2016, 11, (3), 218-230.

22. Desai, S. B.; Madhvapathy, S. R.; Sachid, A. B.; Llinas, J. P.; Wang, Q.; Ahn, G. H.; Pitner, G.; Kim, M. J.; Bokor, J.; Hu, C.; Wong, H.-S. P.; Javey, A. Science 2016, 354, (6308), 99-102.

23. Jaramillo, T. F.; Jorgensen, K. P.; Bonde, J.; Nielsen, J. H.; Horch, S.; Chorkendorff, I. Science 2007, 317, (5834), 100-102.

24. Zhang, J.; Wu, J.; Guo, H.; Chen, W.; Yuan, J.; Martinez, U.; Gupta, G.; Mohite, A.; Ajayan, P. M.; Lou, J. Adv. Mater. 2017, 29, (42).

25. $\quad$ Sun, Y.; Gao, S.; Lei, F.; Xie, Y. Chemical Society Reviews 2015, 44, (3), 623-636.

26. Kong, D.; Wang, H.; Cha, J.; Pasta, M.; Koski, K. J.; Yao, J.; Cui, Y. Nano Lett. 2013, 13, (3), $1341-1347$.

27. Kibsgaard, J.; Chen, Z.; Reinecke, B. N.; Jaramillo, T. F. Nat. Mater. 2012, 11, (11), 963-969.

28. Wang, H.; Lu, Z.; Xu, S.; Kong, D.; Cha, J. J.; Zheng, G.; Hsu, P.-C.; Yan, K.; Bradshaw, D.; Prinz, F. B.; Cui, Y. P. Natl. Acad. Sci. 2013, 110, (49), 19701-19706.

29. $\quad$ Luo, Z.; Ouyang, Y.; Zhang, H.; Xiao, M.; Ge, J.; Jiang, Z.; Wang, J.; Tang, D.; Cao, X.; Liu, C.; Xing, W. Nat. Commun. 2018, 9.

30. Tsai, C.; Li, H.; Park, S.; Park, J.; Han, H.; Norskov, J. K.; Zheng, X.; Abild-Pedersen, F. Nat. Commun. 2017, 8.

31. Leong, W. S.; Li, Y.; Luo, X.; Nai, C. T.; Quek, S. Y.; Thong, J. T. L. Nanoscale 2015, 7, (24), 10823-10831.

32. Bader, R. F. W., Atoms in molecules : a quantum theory. Clarendon Press, Oxford, 1990.

33. Late, D. J.; Liu, B.; Matte, H. S. S. R.; Dravid, V. P.; Rao, C. N. R. ACS Nano 2012, 6, (6), 56355641.

34. Di Bartolomeo, A.; Genovese, L.; Giubileo, F.; Iemmo, L.; Luongo, G.; Foller, T.; Schleberger, M. 2d Mater. 2018, 5, (1).

35. Norskov, J. K.; Bligaard, T.; Logadottir, A.; Kitchin, J. R.; Chen, J. G.; Pandelov, S.; Norskov, J. K. J. Electrochem. Soc. 2005, 152, (3), J23-J26.

36. Hong, J.; Hu, Z.; Probert, M.; Li, K.; Lv, D.; Yang, X.; Gu, L.; Mao, N.; Feng, Q.; Xie, L.; Zhang, J.; Wu, D.; Zhang, Z.; Jin, C.; Ji, W.; Zhang, X.; Yuan, J.; Zhang, Z. Nat. Commun. 2015, 6.

37. Zhou, W.; Zou, X.; Najmaei, S.; Liu, Z.; Shi, Y.; Kong, J.; Lou, J.; Ajayan, P. M.; Yakobson, B. I.; Idrobo, J. C. Nano Lett. 2013, 13, (6), 2615-2622.

38. Skulason, E.; Tripkovic, V.; Bjorketun, M. E.; Gudmundsdottir, S.; Karlberg, G.; Rossmeisl, J.; Bligaard, T.; Jonsson, H.; Norskov, J. K. J. Phys. Chem. C 2010, 114, (50), 22374-22374.

39. Castellanos-Gomez, A.; Cappelluti, E.; Roldan, R.; Agrait, N.; Guinea, F.; Rubio-Bollinger, G. Adv. Mater. 2013, 25, (6), 899-903.

40. Giannozzi, P.; Baroni, S.; Bonini, N.; Calandra, M.; Car, R.; Cavazzoni, C.; Ceresoli, D.; Chiarotti, G. L.; Cococcioni, M.; Dabo, I.; Dal Corso, A.; de Gironcoli, S.; Fabris, S.; Fratesi, G.; Gebauer, R.; Gerstmann, U.; Gougoussis, C.; Kokalj, A.; Lazzeri, M.; Martin-Samos, L.; Marzari, N.; Mauri, F.; Mazzarello, R.; Paolini, S.; Pasquarello, A.; Paulatto, L.; Sbraccia, C.; Scandolo, S.; Sclauzero, G.; Seitsonen, A. P.; Smogunov, A.; Umari, P.; Wentzcovitch, R. M. J. Phys-Condens Mat. 2009, 21, (39), 395502.

41. Larsen, A. H.; Mortensen, J. J.; Blomqvist, J.; Castelli, I. E.; Christensen, R.; Dulak, M.; Friis, J.; Groves, M. N.; Hammer, B.; Hargus, C.; Hermes, E. D.; Jennings, P. C.; Jensen, P. B.; Kermode, J.; Kitchin, 
J. R.; Kolsbjerg, E. L.; Kubal, J.; Kaasbjerg, K.; Lysgaard, S.; Maronsson, J. B.; Maxson, T.; Olsen, T.; Pastewka, L.; Peterson, A.; Rostgaard, C.; Schiotz, J.; Schutt, O.; Strange, M.; Thygesen, K. S.; Vegge, T.; Vilhelmsen, L.; Walter, M.; Zeng, Z. H.; Jacobsen, K. W. J. Phys-Condens Mat. 2017, 29, (27), 273002. 